\title{
Investigation of energy efficiency of two-way relay-assisted multi-band machine-to-machine communications
}

\author{
Hoang Thien $\operatorname{Van}^{1}$, Vo Tien Anh', Danh Hong L ${ }^{3}$, Chi Duong Thi Kim ${ }^{4}$, Hoang-Sy Nguyen \\ ${ }^{1,2}$ The Saigon International University, Ho Chi Minh City, Vietnam \\ ${ }^{3}$ Van Hien University, Ho Chi Minh City, Vietnam \\ ${ }^{4}$ Thu Dau Mot University, Thu Dau Mot City, Vietnam \\ ${ }^{5}$ Binh Duong University, Binh Duong Province, Vietnam
}

\begin{tabular}{l}
\hline \hline Article Info \\
\hline Article history: \\
Received Aug 26, 2020 \\
Revised Jul 12, 2021 \\
Accepted Jul 18, 2021
\end{tabular}

Keywords:

Energy efficiency

Machine-to-machine

Outage probability

Relaying network

Three-time-slot

\section{Corresponding Author:}

Chi Duong Thi Kim

Thu Dau Mot University

Thu Dau Mot City, Binh Duong Province, Vietnam

Email: chidtk@tdmu.edu.vn

\begin{abstract}
In this paper, we improve the uplink energy efficiency (EE) of the multi-band machine-to-machine (M2M) communications underlaying cellular networks. In particular, based on the theory of stochastic geometry, we derive the closed-form expressions of the outage probability (OP), and the average energy efficiency of cellular and Machine-to-Machine users in two-way cooperative relaying networks with three-time-slot setting. We ensure the quality of service (QoS) by considering the OP and the average energy efficiency of all links. It is concluded that the three-time-slot relay-aided Machine-to-Machine communication can offer considerably high QoS and low transmission power for fairly distant machine-to-machine networks.
\end{abstract}

This is an open access article under the CC BY-SA license.

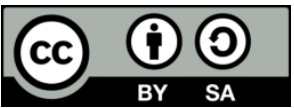

\section{INTRODUCTION}

Machine-to-machine (M2M) communication has been emerging as a promising networking technique for internet of things (IoT) applications in transportation, security and environment [1]. In order to further develop this machine type communication (MTC) in the context of the upcoming fifth generation (5G) communication, research effort has been paid on its foundational device-to-device (D2D) communication [2]. In fact, D2D communication underlaying cellular networks has drawn significant interest because it can provide better throughput, spectral efficiency (SE), energy efficiency (EE) and notably reduce the signal latency and power comsumption by exploiting the devices' proximity, which were elaborated in [3]-[7]. Liu and Natarajan [8], proposed to optimize the performance of D2D networks, the study on compromising the system parameters was conducted. Besides, authors in [9]-[12] assess the energy efficiency (EE) as a key factor for evaluation of D2D networks. For further improvement, relays have been utilized to assist the data transmission process and were intensively investigated in [13]-[18]. From the studies, it is obvious that the system performance of the considered wireless networks can be significantly boosted even under the variety of channel uncertainties thanks to the existence of intermediate relays.

Thereby, two-way relaying network (TWRN) applied in the context of simultaneous wireless information and power transfer (SWIPT) has attracted several researchers among which are [19]-[22]. Remarkably, in [22], the authors proposed a neural network-based relay selection scheme in the context of 
TWRN SWIPT network. As for the system performance evaluation, beside the EE, another indicator being the outage probability (OP) can be utilized as showcased in [23]-[26]. Additionally, several relaying protocols have been developed in [27]-[29].

Inspired by the above studies, herein, we attempt to maximize the EE of the multi-hop two-way M2M communication underlaying cellular networks in the scenario of three-time-slot (3TS). It is noted that the STP is calculated for every node, hence, the mean of EE is calculated, which is consequently named average energy efficiency (AEE). First of all, we mode the spatial and random distribution of the cellular and M2M users utilizing the poisson point process (PPP), yet each type of user is distributed with different density. Then, we optimize the multiple bands' power while studying the dynamic behavior of the networks assuming that there is no inter-device interference. Having been taken into account the above considerations, in context of the two-way M2M communication networks, we derive the closed-form expression of the OP and the AEE of the M2M users in the relay-aided M2M communications in 3TS mode.

The rest of the paper is organized as: section 2 depicts and elaborates the system model. The problem formulation is presented in section 3. Additionally, section 4 presents the Monte Carlo simulation results and discussion. Conclusion is in section 5.

\section{SYSTEM MODEL}

Herein, we consider a typical multi-band M2M communications underlaying a cellular network in which the cellular and the M2M users share the same uplink frequency resources. As shown in Figure 1, a base station (BS) is in charge of allocating the resources, the network's spectrum is split to $K$ bands, subscripted with $i$ to denote the $i_{t h}$ band, $i=1,2, \ldots, K$. We specifically study the 3TS mode where there are intermediate $\mathrm{M} 2 \mathrm{M}$ users acting as relays to assist the data transmission.

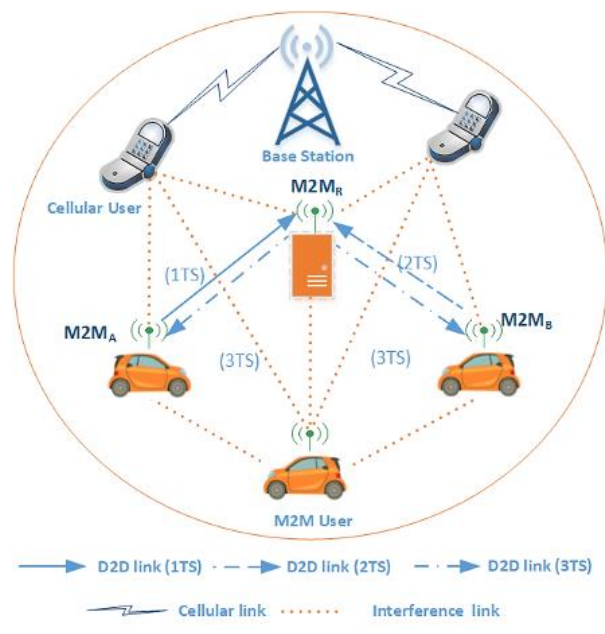

Figure 1. System model

We utilize the stochastic geometry theory to establish the foundation for this study. Firstly, on a 2D plane $\Re$, we distribute the cellular and M2M users in the $i_{t h}$ band, respectively, with homogeneous PPP $\Phi_{c, i}$ and $\Phi_{d, i}$, density $\theta_{c, i}$ and $\theta_{d, i}$. The transmission power of the cellular and M2M users are accordingly $P_{c, i}$ and $P_{d, i}$ which are summed up as.

$$
P_{c}=\sum_{i=1}^{K} P_{c, i},
$$

and

$$
P_{d}=\sum_{i=1}^{K} P_{d, i}
$$

Palm theory states that a typical receiver located at the origin of $\mathfrak{R}$ cannot affect the PPP's characteristics, [30]. Hence, we assign the BS there which works as a receiver for cellular uplink 
transmission and M2M communication assistance. Moreover, we assume the uplink and downlink channels are reciprocal and invariant based on the property of the consecutive equal time slots model.

A two-way DF relay, M2Mr assists a pair of M2M users M2Mato M2M $b$ with power being equal to the M2M power in the $i_{t h}$ band, $P_{r, i}=P_{d, i}$. We distribute these three M2M users with stationary PPP $\Phi_{r, i}$ and density $\theta_{r, i}$. Furthermore, we denote $\mathrm{M} 2 \mathrm{M} a$ to $\mathrm{M} 2 \mathrm{M} r$, and $\mathrm{M} 2 \mathrm{M} r$ to $\mathrm{M} 2 \mathrm{M} b$ distances as $D_{d, i}^{a r}$, and $D_{d, i}^{r b}$, respectively. It is noted that $\mathrm{M} 2 \mathrm{M} a, \mathrm{M} 2 \mathrm{M} b<D_{d, i}$ being the M2Mato M2M $b$ distance. Thereby, we exploit the fraction $\lambda_{1}$ to express the relationship between them as.

$$
\begin{aligned}
& D_{d, i}^{a r}=D_{d, i}\left(\frac{1}{1+\lambda_{1}}\right) \\
& \text { and } \\
& D_{d, i}^{r b}=D_{d, i}\left(\frac{\lambda_{1}}{1+\lambda_{1}}\right)
\end{aligned}
$$

Similar to [30], we consider the impact of Rayleigh fading and path loss in our propagation channel model. In general, to indicate the Rayleigh fading coefficient of the $l-k$ link $(l, k=a, b, r)$, we utilize the denotation $h_{d, i, l k}$ given that $h_{d, i, l k}=h_{d, i, k l}$. For example, the cellular and M2M users receive power of $P_{r x}$ is as shown in (5).

$$
P_{r x}=P_{t x} h D^{-m}
$$

where we have the transmit power $P_{t x}$, the Rayleigh fading coefficient $h$, the distance from the transmitter to its corresponding receiver $D$, and an even-number path-loss exponent $m, m \geq 2$. It is noteworthy that when $\mathrm{M} 2 \mathrm{M}$ communication reuses the cellular resources, it generates interference to the typical receiver. This interference is caused by the cellular, M2M users, and M2M relay users.

\section{PROBLEM FORMULATION}

In this section, we formulate firstly the signal-to-interference-plus-noise ratio (SINR) then the OP performance. Accordingly, we derive the closed-form expression of AEE in the context of multi-band M2M communication for 3TS, then simulate it following Monte Carlos method.

- The signal-to-interfrence-plus-noise ratio

For 3TS mode, in the 1st and 2nd time slots, information is respectively transmitted from M2Ma and $\mathrm{M} 2 \mathrm{M} b$ to $\mathrm{M} 2 \mathrm{Mr} r$, with power $P_{d, i}$. Afterwards, in the 3rd time slot, $\mathrm{M} 2 \mathrm{M} r$ mixes these two information flows, network-codes and sends it back to M2M $a$ and $\mathrm{M} 2 \mathrm{M} b$.

Within the 1 st and 2nd time slots, $\mathrm{M} 2 \mathrm{M} r$ receives signal from respectively $\mathrm{M} 2 \mathrm{M} a$ and $\mathrm{M} 2 \mathrm{M} b$ as;

$$
y_{a r, i}=\sqrt{P_{d, i}\left(\frac{D_{d, 00, i}}{1+\lambda_{1}}\right)^{-m} h_{a r, 00}}+I_{0, i}+N_{0}
$$

and

$$
y_{b r, i}=\sqrt{P_{d, i}\left(\frac{\lambda_{1} D_{d, 00, i}}{1+\lambda_{1}}\right)^{-m} h_{b r, 00}}+I_{0, i}+N_{0}
$$

where the Rayleigh fading channel coefficient $h_{a r, 00}$ and $h_{b r, 00}$ of $\mathrm{M} 2 \mathrm{M} a$ to $\mathrm{M} 2 \mathrm{M} r$ and $\mathrm{M} 2 \mathrm{M} r$ to $\mathrm{M} 2 \mathrm{M} b$ links in the $i_{t h}$ band, and the power of interference caused by the cellular users, the M2M user, and M2Mr to the typical receiver as $I_{0, i}=\sum_{j \in \Phi_{c, i}}\left(P_{c, i} h_{c, j 0} D_{c, j 0, i}^{-m}\right)^{-1 / 2}+\sum_{l \in \Phi_{d, i}}\left(P_{d, i} h_{d, l 0} D_{d, l 0, i}^{-m}\right)^{-1 / 2}+$ $\sum_{k \in \Phi_{r, i}}\left(P_{d, i} h_{r, k 0} D_{r, k 0, i}^{-m}\right)^{-1 / 2}$.

Within the 3rd time slot, we have a portion $\lambda_{2},\left(0<\lambda_{2}<1\right)$ of the transmission power at $\mathrm{M} 2 \mathrm{Mr}$, $P_{d, i}$, used for transmitting the mixed flow of signal back to M2M $a$ and M2M $b$. In particular, we allocate $\lambda_{2} P_{d, i}$ for the $\mathrm{M} 2 \mathrm{M} r$ to $\mathrm{M} 2 \mathrm{M} a$ link, and $\left(1-\lambda_{2}\right) P_{d, i}$ for the $\mathrm{M} 2 \mathrm{M} r$ to $\mathrm{M} 2 \mathrm{M} b$ link. Thus, M2M $a$ and $\mathrm{M} 2 \mathrm{M} b$, respectively, receive signal of.

$$
y_{r a, i}=\sqrt{\lambda_{2} P_{d, i}\left(\frac{D_{d, 00, i}}{1+\lambda_{1}}\right)^{-m} h_{r a, 00}}+I_{0, i}+N_{0},
$$

Investigation of energy efficiency of two-way relay-assisted multi-band machine-to- .... (Hoang Thien Van) 
and

$$
y_{r b, i}=\sqrt{\left(1-\lambda_{2}\right) P_{d, i}\left(\frac{\lambda_{1} D_{d, 00, i}}{1+\lambda_{1}}\right)^{-m} h_{r b, 00}}+I_{0, i}+N_{0} .
$$

Then, as we eliminate the noise term from SINR, the signal-to-interference ratio (SIR) in the $i_{t h}$ band of M2M $a$ and M2M $b$ via DF-operated M2Mr can be obtained from (10) and (11).

$$
\gamma_{a, i}=\min \left(\gamma_{a r, i}, \gamma_{b r, i}, \gamma_{r a, i}\right)
$$

and

$$
\gamma_{b, i}=\min \left(\gamma_{a r, i}, \gamma_{b r, i}, \gamma_{r b, i}\right)
$$

where concerning the Palm theory [30], the instantaneous SIR of the 1st, 2nd, and 3rd time slots are shown in (12).

$$
\gamma_{a r, i}=\frac{P_{d, i} h_{a r, 00}}{I_{0, i}}\left(\frac{1}{1+\lambda_{1}} D_{d, 00, i}\right)^{-m}
$$

and

$$
\gamma_{b r, i}=\frac{P_{d, i} h_{b r, 00}}{I_{0, i}}\left(\frac{\lambda_{1}}{1+\lambda_{1}} D_{d, 00, i}\right)^{-m}
$$

and

$$
\gamma_{r a, i}=\frac{\lambda_{2} P_{d, i} h_{r a, 00}}{I_{0, i}}\left(\frac{1}{1+\lambda_{1}} D_{d, 00, i}\right)^{-m}
$$

and

$$
\gamma_{r b, i}=\frac{\left(1-\lambda_{2}\right) P_{d, i} h_{r b, 00}}{I_{0, i}}\left(\frac{\lambda_{1}}{1+\lambda_{1}} D_{d, 00, i}\right)^{-m}
$$

- The outage probability of typical receivers

This OP specifies the probability that the instantaneous SIR recorded at the receivers drops below the threshold value of $\gamma_{t h}$. Hence, it is possible for us to use the cumulative distribution function (CDF) of the received SIR to formulate the OP, as described in the Theorem 1. Theorem 1 .

The OP of the typical M2M receivers via DF-operated M2M $r$ at M2M $a$ and $\mathrm{M} 2 \mathrm{M} b$ in the $i_{t h}$ band, can be obtained respectively from (16) and (17).

$$
\operatorname{Pr}\left(\gamma_{a, i}<\gamma_{t h}\right)=1-e^{-\Psi_{a, i}\left[\theta_{d, i}+\theta_{r, i}+\theta_{c, i}\left(\frac{P_{c, i}}{P_{d, i}}\right)^{\frac{2}{m}}\right]}
$$

and

$$
\operatorname{Pr}\left(\gamma_{b, i}<\gamma_{t h}\right)=1-e^{-\Psi_{b, i}\left[\theta_{d, i}+\theta_{r, i}+\theta_{c, i}\left(\frac{P_{c, i}}{P_{d, i}}\right)^{\frac{2}{m}}\right]}
$$

where $\quad \Psi_{a, i}=\pi \gamma_{t h}^{\frac{2}{m}} \Gamma\left(1+\frac{2}{m}\right) \Gamma\left(1-\frac{2}{m}\right)\left(\frac{D_{d, 00, i}}{1+\lambda_{1}}\right)^{2}\left(1+\lambda_{1}^{2}+\lambda_{2}^{-\frac{2}{m}}\right) \quad, \quad$ and $\quad \Psi_{b, i}=\pi \gamma_{t h}^{\frac{2}{m}} \Gamma\left(1+\frac{2}{m}\right) \Gamma$ $\left(1-\frac{2}{m}\right)\left(\frac{D_{d, 00, i}}{1+\lambda_{1}}\right)^{2}\left(1+\lambda_{1}^{2}+\lambda_{1}^{2}\left(1-\lambda_{2}\right)^{-\frac{2}{m}}\right)$.

Proof: 
As aforementioned in the network model section, $h_{a r, 00}$ is independently and exponentially distributed with unit mean. As the study is conducted on 2D planes and $\gamma_{a r, i}$ follows the independent exponential distribution of M2M users and cellular channel gains, with regard to the (12). Based on the Laplace transform definition and stochastic geometry theory in [31], the OP in the 1st time slot can be derived as.

$$
\operatorname{Pr}\left\{\gamma_{a r, i}<\gamma_{t h}\right\}=1-e^{-\Psi_{a r, i}\left[\theta_{d, i}+\theta_{r, i}+\theta_{c, i}\left(\frac{P_{c, i}}{P_{d, i}}\right)^{\frac{2}{m}}\right]}
$$

where $\Gamma(x)=\int_{0}^{\infty} e^{-t} t^{x-1} d t$, and $\Psi_{a r, i}=\pi \gamma_{t h}^{\frac{2}{m}}\left(\frac{D_{d, 00, i}}{1+\lambda_{1}}\right)^{2} \Gamma\left(1+\frac{2}{m}\right) \Gamma\left(1-\frac{2}{m}\right)$.

Similarly, based on (13), the OP in the 2 nd time slot is given as;

$$
\operatorname{Pr}\left\{\gamma_{b r, i}<\gamma_{t h}\right\}=1-e^{-\Psi_{b r, i}\left[\theta_{d, i}+\theta_{r, i}+\theta_{c, i}\left(\frac{P_{c, i}}{P_{d, i}}\right)^{\frac{2}{m}}\right]}
$$

where $\Psi_{b r, i}=\pi \gamma_{t h}^{\frac{2}{m}}\left(\frac{\lambda_{1} D_{d, 00, i}}{1+\lambda_{1}}\right)^{2} \Gamma\left(1+\frac{2}{m}\right) \Gamma\left(1-\frac{2}{m}\right)$.

In the 3rd time slot, using (14), the OP at $\mathrm{M}_{2} \mathrm{M}_{a}$ and $\mathrm{M}_{2} \mathrm{M}_{b}$ are respectively formulated as;

$$
\operatorname{Pr}\left\{\gamma_{r a, i}<\gamma_{t h}\right\}=1-e^{-\Psi_{r a, i}\left[\theta_{d, i}+\theta_{r, i}+\theta_{c, i}\left(\frac{P_{c, i}}{P_{d, i}}\right)^{\frac{2}{m}}\right]}
$$

and

$$
\operatorname{Pr}\left\{\gamma_{r b, i}<\gamma_{t h}\right\}=1-e^{-\Psi_{r b, i}\left[\theta_{d, i}+\theta_{r, i}+\theta_{c, i}\left(\frac{P_{c, i}}{P_{d, i}}\right)^{\frac{2}{m}}\right]}
$$

where $\quad \Psi_{r a, i}=\pi \Gamma\left(1+\frac{2}{m}\right) \Gamma\left(1-\frac{2}{m}\right)\left(\frac{1}{1+\lambda_{1}} D_{d, 00, i}\right)^{2}\left(\frac{\gamma_{t h}}{\lambda_{2}}\right)^{\frac{2}{m}} \quad, \quad$ and $\quad \Psi_{r b, i}=\pi \Gamma\left(1+\frac{2}{m}\right)$ $\Gamma\left(1-\frac{2}{m}\right)\left(\frac{\lambda_{1}}{1+\lambda_{1}} D_{d, 00, i}\right)^{2}\left(\frac{\gamma_{t h}}{1-\lambda_{2}}\right)^{\frac{2}{m}}$.

Subsequently, using (18), (19), (20), (21) to combine with (10), (11), after some basic algebraic manipulations, the OP based on SIR at UED $a$ and UED $b$ is obtained in (16) and (17), respectively. The proof for Theorem 1 ends here.

- Average energy efficiency of typical receivers

Based on Theorem 1, we can formulate the average energy efficiency (AEE) of the typical receiver $\mathrm{M} 2 \mathrm{M}$ user in 3TS mode in the $K$ bands respectively at $\mathrm{M} 2 \mathrm{M} a$ and $\mathrm{M} 2 \mathrm{M} b$ in the $i_{t h}$ band as.

$$
A E E_{a, i}=\sum_{i=1}^{K} \frac{W_{i} \log _{2}\left(1+\gamma_{t h}\right)}{P_{d, i}} e^{-\Psi_{a, i}^{3 T S}\left(\theta_{d, i}+\theta_{r, i}+\theta_{c, i}\left(\frac{P_{c, i}}{P_{d, i}}\right)^{\frac{2}{m}}\right)},
$$

and

$$
A E E_{b, i}=\sum_{i=1}^{K} \frac{W_{i} \log _{2}\left(1+\gamma_{t h}\right)}{P_{d, i}} e^{-\Psi_{b, i}^{3 T S}\left(\theta_{d, i}+\theta_{r, i}+\theta_{c, i}\left(\frac{P_{c, i}}{P_{d, i}}\right)^{\frac{2}{m}}\right)} .
$$

where $\Psi_{a, i}^{3 T S}$ and $\Psi_{b, i}^{3 T S}$ described in Theorem 1. In the next section, we present the simulation results of the $\mathrm{OP}$ and the AEE. 


\section{NUMERICAL SIMULATION}

Using Matlab, we obtained the Monte Carlo simulation results for the OP and the AEE of the in-studied M2M system for M2Ma and $\mathrm{M} 2 \mathrm{M} b$. We used the following system parameters in [32] show Table 1. Figure 2 and Figure 3 present the OP performance at $\mathrm{M} 2 \mathrm{M} a$ and $\mathrm{M} 2 \mathrm{M} b$, respectively, versus the M2M user density. It can be observed that the M2Ma curves originate at a slightly higher OP level than $\mathrm{M} 2 \mathrm{M} b$ ones. Nevertheless, as the M2M users become denser, the OP increases and all the curves rapidly rise to approximately $100 \%$ probability of shutting down the systems. Besides, lower $P_{c, i}$ results in lower OP meaning that the system performance is better.

Moreover, Figure 4 and Figure 5 describe the AEE performance at $\mathrm{M} 2 \mathrm{M} b$ and $\mathrm{M} 2 \mathrm{M} b$, respectively, versus the M2M user density. One of the obvious traits is that at the lowest level of $P_{c, i}$, the systems perform the best as the AEE curves in these cases are the highest. As for Figure 4, all the AEE curves increase and peak at the points where there are $4 \mathrm{M} 2 \mathrm{M}$ users perm ${ }^{2}$, then decrease to approximately the same levels as their original values. Besides, in Figure 5, the curves follow similar trends as the curves in Figure 4 but with the highest AEE values reached at $7 \mathrm{M} 2 \mathrm{M}$ users per $\mathrm{m}^{2}$. With regards to the two former figures, we can claim that the M2M user density raises in association with the increase in the OP and the increase in the AEE (only up to some certain values). This is because as there are more M2M users, the energy will be used more efficiently. However, if there are too many users, they will introduce more severe inter-device interference to the networks leading to the higher probability of system shutdown and considerable AEE downgrade.

Table 1. The main parameters

\begin{tabular}{lc}
\multicolumn{1}{c}{ Parameters } & Values \\
\hline The number of bands & $K=5$ \\
The ith bandwidth, & $W_{i}=20(\mathrm{MHz})$ \\
The transmission power of cellular & $P_{c, i}=125(\mathrm{~mW})$ \\
The transmission power of M2M users & $P_{d, i}=60(\mathrm{~mW})$ \\
The power allocation at M2Mr, & $\lambda_{2}=0.5$ \\
$\lambda_{1}=0.5$ \\
The fraction of distances between M2Mr and M2Ma, M2Mb & $\zeta_{c, i}=\zeta_{d, i}=0(\mathrm{~dB})$ \\
The threshold SIR of cellular and M2M users & $\varepsilon_{c, i}=\varepsilon_{d, i}=0.05$ \\
The threshold OP of cellular and M2M users & $P_{d, i, u p}=20(\mathrm{~mW})$ \\
The threshold transmission power of M2M users & $m=4$ \\
The path-loss coefficient & {$[50,60,70,80,90]$} \\
The cellular link distances $\left[D_{c, 00,1}, D_{c, 00,2}, \cdots, D_{c, 00, K}\right]$ & {$[10,20,30,20,10]$} \\
The M2M link distances $\left[D_{d, 00,1}, D_{d, 00,2}, \cdots, D_{d, 00, K}\right]$ & {$[10,1,10,10,10] \times 10^{-5}$} \\
The cellular user density $\left[\theta_{c, 1}, \theta_{c, 2}, \cdots, \theta_{c, K}\right]$ & {$[10,1,10,10,10] \times 10^{-4}$} \\
The M2M user density, $\left[\theta_{d, 1}, \theta_{d, 2}, \cdots, \theta_{d, K}\right]$ & {$[10,1,10,10,10] \times 10^{-4}$} \\
The potential two-way M2Mr density, $\left[\theta_{r, 1}, \theta_{r, 2}, \cdots, \theta_{d, K}\right]$ & \\
\hline
\end{tabular}

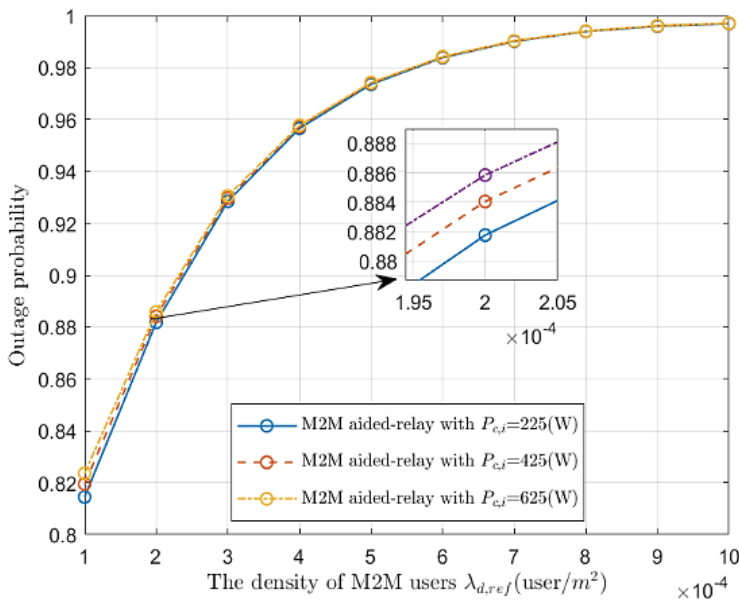

Figure 2. OP performance at M2Ma versus M2M user density

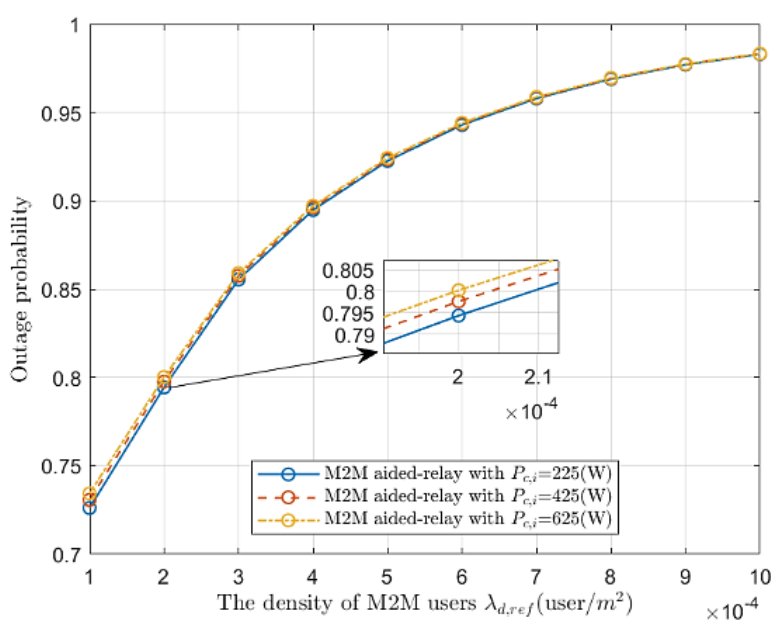

Figure 3. OP performance at M2Mb versus M2M user density 


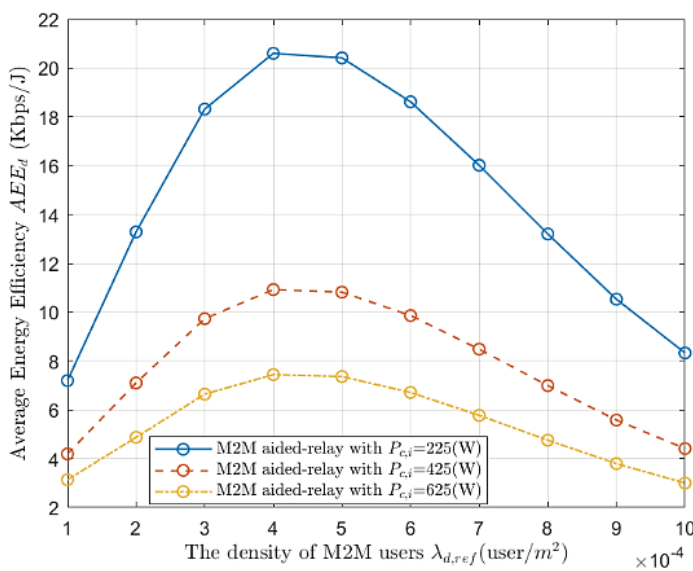

Figure 4. AEE performance at M2Ma versus M2M user density

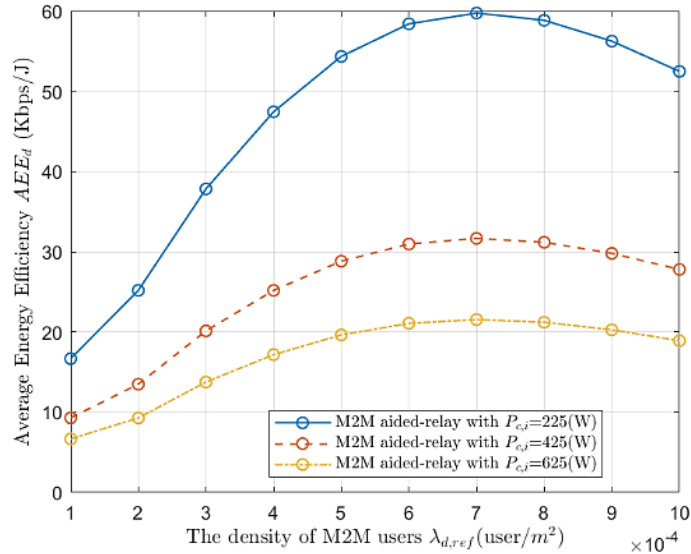

Figure 5. AEE performance at M2Mb versus M2M user density

\section{CONCLUSION}

To conclude, in this study, we analyze the OP and the AEE of the multi-hop M2M communication underlaying cellular networks in 3TS mode. Based on simulation results, readers can compromise the M2M user density and the transmission power of the cellular users for optimal values of the OP and the AEE. It should be noted that for such cooperative relaying networks, the presence of many M2M users does not always optimize the performance of the systems. For any replicating study, $P_{c, i}=225 \mathrm{~W}$ will be a good starting value for the evaluation. In the upcoming research pieces, we will investigate how to minimize the cross-tier interference caused by the cellular and M2M users with the help of power allocation and band selection.

\section{ACKNOWLEDGEMENTS}

Thanks for the Saigon International University (SIU) funds supporting this project.

\section{REFERENCES}

[1] S. N. Swain, R. Thakur and S. R. M. Chebiyyam, "Coverage and Rate Analysis for Facilitating Machine-toMachine Communication in LTE-A Networks Using Device-to-Device Communication," IEEE Transactions on Mobile Computing, vol. 16, no. 11, pp. 3014-3027, Nov. 2017, doi: 10.1109/TMC.2017.2684162.

[2] G. Rigazzi, N. K. Pratas, P. Popovski and R. Fantacci "Aggregation and trunking of M2M traffic via D2D connections," in Proc. IEEE Int. Conf. Communications, Jun. 2015, pp. 2973-2978, doi: 10.1109/ICC.2015.7248779.

[3] L. Wei, R. Q. Hu, Y. Qian and G. Wu, "Enable device-to-device communications underlaying cellular networks: challenges and research aspects," IEEE Communications Magazine, vol. 52, no. 6, 2014, pp. 90-96, doi: 10.1109/MCOM.2014.6829950.

[4] S. Wang, W. Guo, Z. Zhou, Y. Wu and X. Chu, "Outage probability for multi-hop D2D communications with shortest path routing," IEEE Communications Letter, vol. 19, no. 11, pp. 1997-2000, Nov. 2015, doi: 10.1109/LCOMM.2015.2475428.

[5] X. Lin, J. G. Andrews, A. Ghosh and R. Ratasuk, "An overview of 3GPP device-to-device proximity services," IEEE Communications Magazine, vol. 52, no. 4, pp. 40-48, 2014, doi: 10.1109/MCOM.2014.6807945.

[6] J. Liu, N. Kato, J. Ma and N. Kadowaki, "Device-to-device communication in LTE-advanced networks: a survey," IEEE Communications Surveys \& Tutorials, vol. 17, no. 4, pp. 1923-1940, 2015, doi: 10.1109/COMST.2014.2375934.

[7] O. Bello and S. Zeadally, "Intelligent device-to-device communication in the internet of things," IEEE Systems Journal, vol. 10, no. 3, pp. 1172-1182, 2016, doi: 10.1109/JSYST.2014.2298837.

[8] C. Liu and B. Natarajan, "Power-aware maximization of ergodic capacity in D2D underlay networks," IEEE Transactions on Vehicular Technology, vol. 66, no. 3, pp. 2727-2739, 2017, doi: 10.1109/TVT.2016.2583960.

[9] L. Wei, R. Q. Hu, Y. Qian and G. Wu, "Energy efficiency and spectrum efficiency of multi hop device-to-device communications underlaying cellular networks," IEEE Transactions on Vehicular Technology, vol. 65, no. 1, pp. 367-380, Jan. 2016, doi: 10.1109/TVT.2015.2389823.

[10] M. Sheng, Y. Li, X. Wang, J. Li and Y. Shi, "Energy Efficiency and Delay Tradeoff in Device-to-Device Communications Underlaying Cellular Networks," IEEE Journal on Selected Areas in Communications, vol. 34, no. 1, pp. 92-106, 2016, doi: 10.1109/JSAC.2015.2471395.

[11] Z. Zhou, M. Dong, K. Ota, J. Wu and T. Sato, "Energy Efficiency and Spectral Efficiency Tradeoff in Device-toDevice (D2D) Communications," IEEE Wireless Communications Letters, vol. 3, no. 5, pp. 485-488, 2014, doi: 10.1109/LWC.2014.2337295. 
[12] J. M. B. da Silva, G. Fodor and T. F. Maciel, "Performance analysis of network-assisted two-hop D2D communications," In Proc. IEEE Globecom Workshops (GC Wkshps), Austin, TX, 2014, pp. 1050-1056, doi: 10.1109/GLOCOMW.2014.7063572.

[13] X. Liang, S. Jin, X. Gao and K. K. Wong, "Outage performance for decode-and-forward two-way relay network with multiple interferers and noisy relay," IEEE Transactions on Communications, vol. 61, no. 2, pp. 521-531, 2013, doi: 10.1109/TCOMM.2012.122112.110778.

[14] P. K. Upadhyay and P. K. Sharma, "Max-Max User-Relay Selection Scheme in Multiuser and Multirelay Hybrid Satellite-Terrestrial Relay Systems," IEEE Communications Letters, vol. 20, no. 2, pp. 268-271, Feb. 2016, doi: 10.1109/LCOMM.2015.2502599.

[15] Z. Hasan, H. Boostanimehr and V. K. Bhargava, "Green cellular networks: a survey, some research issues and challenges," IEEE Communications Surveys \& Tutorials, vol. 13, no. 4, pp. 524-540, 2011, doi: 10.1109/SURV.2011.092311.00031.

[16] M. Rehman, P. Nallagownden and Z. Baharudin, "Efficiency investigation of SS and SP compensation topologies for wireless power transfer," International Journal of Power Electronics and Drive Systems (IJPEDS), vol. 10, pp. 2157-2164, 2019, doi: 10.11591/ijpeds.v10.i4.pp2157-2164.

[17] A. K. Obais and A. F. Ruzij, "Design and implementation of an efficient WPT system," International Journal of Power Electronics and Drive Systems, vol. 11, no. 2, pp. 711-725, 2020, doi: 10.11591/ijpeds.v11.i2.pp711-725.

[18] M. Hasan and E. Hossain, "Distributed resource allocation for relay-aided device-to-device communication under channel uncertainties: a stable matching approach," IEEE Transactions on Communications, vol. 63, no. 10, pp. 3882-3897, 2015, doi: 10.1109/TCOMM.2015.2466238.

[19] V. N. Vo, T. G. Nguyen, C. S. In, Z. A. Baig and S. Sanguanpong, "Secrecy Outage Performance Analysis for Energy Harvesting Sensor Networks with a Jammer Using Relay Selection Strategy," in IEEE Access, vol. 6, pp. 23406-23419, 2018, doi: 10.1109/ACCESS.2018.2829485.

[20] K. Tutuncuoglu, B. Varan and A. Yener, "Throughput maximization for two-way relay channels with energy harvesting nodes: the impact of relaying strategies," IEEE Transactions on Communications, vol. 63, no. 6, pp. 2081-2093, 2015, doi: 10.1109/TCOMM.2015.2427162.

[21] H. S. Nguyen, M. Voznak, M. T. Nguyen and L. Sevcik, "Performance analysis with wireless power transfer constraint policies in full-duplex relaying networks," Elektron Elektrotech, vol. 23, no. 4, pp. 70-76, 2017, doi: $10.5755 /$ j01.eie.23.4.18725.

[22] Z. Zhang, Y. Lu, Y. Huang and P. Zhang, "Neural Network-Based Relay Selection in Two-Way SWIPT-Enabled Cognitive Radio Networks," in IEEE Transactions on Vehicular Technology, vol. 69, no. 6, pp. 6264-6274, 2020, doi: 10.1109/TVT.2020.2984327.

[23] Y. Ni, S. Jin, K. K. Wong, H. Zhu and S. Shao, "Outage performances for device-to-device communication assisted by two-way amplify-and-forward relay protocol," In Proc. IEEE Wireless Communications and Networking Conference (WCNC), Istanbul, 2014, pp. 502-507, doi: 10.1109/WCNC.2014.6952079.

[24] N. T. Do, D. B. D. Costa, T. Q. Duong and B. An, "A BNBF user selection scheme for NOMA-based cooperative relaying systems with SWIPT," IEEE Communications Letters, vol. 21, no. 3, pp. 664-667, 2017, doi: 10.1109/LCOMM.2016.2631606.

[25] H. S. Nguyen, T. T. H. Ly, T. S. Nguyen, V. V. Huynh, T. L. Nguyen and M. Voznak, "Outage performance analysis and SWIPT optimization in energy-harvesting wireless sensor network deploying NOMA," Sensors, vol. 619, no. 3, p. 613, 2019, doi: 10.3390/s19030613.

[26] X. Zhang, Z. Zhang, J. Xing, R. Yu, P. Zhang, and W. Wang,"Exact outage analysis in cognitive two-way relay networks with opportunistic relay selection under primary user's interference," IEEE Transactions on Vehicular Technology, vol. 64, no. 6, pp. 2502-2511, 2015, doi: 10.1109/TVT.2014.2346615.

[27] H. S. Nguyen, D. T. Do and M. Voznak, "Two-way relaying networks in green communications for 5G: optimal throughput and tradeoff between relay distance on power splitting-based and time switching-based relaying SWIPT," AEU - International Journal of Electronics and Communications, vol. 70, no. 12, pp.1637-1644, 2016, doi: 10.1016/j.aeue.2016.10.002.

[28] H. S. Nguyen, D. T. Do, A. H. Bui and M. Voznak, "Self-powered wireless two-way relaying networks: model and throughput performance with three practical schemes," Wireless Pers. Commun., vol. 97, no, 11, pp. 1-9, 2017, doi 10.1007/s11277-017-4526-3.

[29] N. Nomikos, T. Charalambous, I. Krikidis, D. N. Skoutas, D. Vouyioukas and M. Johansson, "A buffer-aided successive opportunistic relay selection scheme with power adaptation and inter-relay interference cancellation for cooperative diversity systems," IEEE Transactions on Communications, vol. 63, no. 5, pp. 1623-1634, 2015, doi: 10.1109/TCOMM.2015.2416234.

[30] F. Baccell and B. Blaszczyszyn, "Stochastic geometry and wireless networks volume I: theory," NOW: Foundations and Trends in Networking, vol. 33, 2010.

[31] M. Haenggi, Stochastic geometry for wireless networks, Cambridge, UK: Cambridge University Press2012.

[32] Y. Jiang, Q. Liu, F. Zheng, X. Gao and X. You, "Energy-efficient joint resource allocation and power control for D2D communications," IEEE Trans. Veh. Technol., vol. 65, pp. 6119-6127, 2016, doi: 10.1109/TVT.2015.2472995. 